\title{
Influences of Fascicle Length During Isometric Training on Improvement of Muscle Strength
}

\section{AUTHOR(S):}

Tanaka, Hiroki; Ikezoe, Tome; Umehara, Jun; Nakamura, Masatoshi; Umegaki, Hiroki; Kobayashi, Takuya; Nishishita, Satoru; Fujita, Kosuke; Araki, Kojiro; Ichihashi, Noriaki

\section{CITATION:}

Tanaka, Hiroki ... [et al]. Influences of Fascicle Length During Isometric Training on Improvement of Muscle Strength. Journal of Strength and Conditioning Research 2016, 30(11): 3249-3255

\section{ISSUE DATE:}

2016-11-01

URL:

http://hdl.handle.net/2433/231302

\section{RIGHT:}

(c) 2016 by the National Strength \& Conditioning Association. This is a non-final version of an article published in final form in Journal of Strength and Conditioning Research. 30(11):3249-3255, NOV 2016.; The full-text file will be made open to the public on 01 November 2017 in accordance with publisher's 'Terms and Conditions for Self-Archiving'.; $こ の$ 論文は出版社版でありません。引用の際には出版社版をご確認ご利用ください。; This is not the published version.

Please cite only the published version. 
2 Hiroki Tanaka ${ }^{1 *}$, Tome Ikezoe ${ }^{1}$, Jun Umehara ${ }^{1}$, Masatoshi Nakamura ${ }^{1}{ }^{2}$, Hiroki Umegaki ${ }^{1}$,

3 Takuya Kobayashi ${ }^{1}$, Satoru Nishishita ${ }^{1}$, Kosuke Fujita ${ }^{1}$, Kojiro Araki ${ }^{1}$, Noriaki Ichihashi ${ }^{1}$

1) Human Health Sciences, Graduate School of Medicine, Kyoto University, 53, Shogoin-Kawahara-

6 cho, Sakyo-ku, Kyoto 606-8507, Japan.

2) Department of Faculty of Health and Sports Science, Doshisha University, Kyoto, Japan

*Corresponding author:

Hiroki Tanaka $(\bowtie)$

Kyoto 606-8507,

Japan.

E-mail: tanaka.hiroki.8w@kyoto-u.ac.jp 


\section{$1 \quad$ Abstract}

2 This study investigated whether low-intensity isometric training would elicit a greater improvement

3 in maximum voluntary contraction (MVC) at the same fascicle length, rather than the joint angle,

4 adopted during training. Sixteen healthy women (21.8 \pm 1.5 years) were randomly divided into an

5 intervention group and a control group. Before (Pre-) and after (Post-) training, isometric

6 plantarflexion MVCs were measured every $10^{\circ}$ through the range of ankle joint position from

$720^{\circ}$ dorsiflexion to $30^{\circ}$ plantarflexion (i.e. 6 ankle angles). Medial gastrocnemius (MG) fascicle length

8 was also measured at each positions, using B-mode ultrasound under 3 conditions of muscle activation:

9 at rest, 30\%MVC at respective angles, and MVC. Plantarflexion resistance training at an angle of $20^{\circ}$ plantarflexion was performed 3 days a week for 4 weeks at 30\%MVC using 3 sets of twenty, 3 seconds (-s) isometric contractions. MVC in the intervention group increased at $0^{\circ}$ and $10^{\circ}$ plantarflexion ( $0^{\circ}$; Pre $81.2 \pm 26.5$ Nm, Post $105.0 \pm 21.6$ Nm, 10; Pre $63.0 \pm 23.6$ Nm, Post $81.3 \pm$ 20.3 Nm), which was not the angle used in training $\left(20^{\circ}\right)$. However, the fascicle length adopted in training at $20^{\circ}$ plantarflexion and $30 \% \mathrm{MVC}$ was similar to the value at $0^{\circ}$ or $10^{\circ}$ plantarflexion at MVC. Low-intensity isometric training at a shortened muscle length may be effective for improving MVC at a lengthened muscle length because of specificity of the fascicle length than the joint angle.

18 Keywords: Strength, Isometric training, Low-intensity training, Specificity of fascicle length, 
1 Specificity of joint angle $\cdot$ Ankle plantarflexion

2

3 
1

2

3

4

5

6

7

9

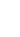

\section{INTRODUCTION}

It is well known that greater effects of resistance training on muscle strength, both with concentric

or eccentric contractions, are observed under the conditions used during training, such as the same movement speed (9) or the same movement pattern (19). When resistance training is performed under isometric conditions, strength gains are known to be influenced by the specificity of the joint angle used during training $(10,13)$. Kitai and Sale (10) reported that isometric training of ankle plantarflexion using maximum voluntary contraction (MVC) at an angle of $0^{\circ}$ (i.e., $90^{\circ}$ angle between the tibia and sole of foot) produced improvement in MVC only around $0^{\circ}$ of ankle position (MVC between $-5^{\circ}$ and $5^{\circ}$ ). These results demonstrate the 'so called' specificity of joint angle on training effects. However, Weir et al. (20, 21) reported that isometric training of knee extension at an intensity of $80 \% \mathrm{MVC}$ and at a $45^{\circ}$ knee position angle resulted in significant improvement in MVC over a range of $30^{\circ}$ around the training angle. Rasch and Pierson (18) similarly reported that isometric training of elbow flexion at an intensity of $100 \% \mathrm{MVC}$ and at a $90^{\circ}$ elbow angle produced significant improvement in MVC over a range of $30^{\circ}$ around the training angle. Thus, contrary to the specificity of joint angle in isometric training previously accepted, some reports have now demonstrated that improvement in MVC can be obtained over a wide range of joint angles around the training angle. The reason for this inconsistency, however, has not been clearly elucidated. 
1

2

Recent studies examining the changes in the muscle fascicle length during isometric contraction, using ultrasound imaging, have shown the fascicle length to be influenced by both the joint angle and the force exerted during an isometric contraction $(3,8)$. In particular, these two studies $(3,8)$ demonstrated that the fascicle length is dependent on the contraction levels, even if a constant joint angle is used during isometric contraction. From these facts, we hypothesized that "specificity" in isometric training, which results in the maximum improvement of MVC, may depend not on the joint angle but, rather, on the fascicle length during training. Several previous studies $(18,20,21)$ found improvements of MVC over wide range of joint angles including the training angle, which indicates that the training effect did not strictly follow the specificity of joint angle. In these studies, highintensity and long-duration isometric contraction or a short rest time was adopted. For instance, the training protocol used in Rasch and Pierson (18) was $15 \mathrm{~s}$ (long duration) of MVC, and it was 80\%MVC with $30 \mathrm{~s}$ (short) rest time in Weir et al. $(20,21)$. Therefore, it could be predicted that fatigue would occur during training, and the contraction may not have been maintained throughout training, which means that actual contraction during training may have been performed at various fascicle length in these studies $(18,20,21)$. Taking these previous results into account, there is a possibility that the underlying cause of the wider range of joint angles in strength improvement following isometric training may be that the specificity of strength improvement may owe to the fascicle length during training rather than the joint angle. 
1 Arampatzis et al. (3) indicated that the fascicle length measured during low-intensity contraction at

2 a joint position in which the muscle is in a shortened length was equal to the fascicle length measured

3 during a high-intensity contraction at a joint position in which the muscle is in an extended length. In

4 order to discriminate the difference of specificity in training between the fascicle length and the joint

5 angle, we conducted this study by investigating the effect of low-intensity training at shortened muscle

6 length and examine whether the MVC improvement occurs at the same fascicle length or at the same

7 joint angle.

8 The purpose of the present study was to examine whether low-intensity isometric training would

9 yield a greater improvement in MVC at the same fascicle length as the training condition. We

hypothesized that low-intensity isometric training would have a greater improvement in MVC at the

11 same fascicle length, rather than at the same joint angle, used during training.

METHODS

15 Experimental Approach to the Problem

17 The experimental design of this study was a randomized controlled trial. A flowchart of the 
1

2

3

4

5

6

7

9

were familiarized with the MVC procedure for plantarflexion. In the initial week of the experiment, subjects attended 3 familiarization sessions, practicing MVCs at the 6 ankle joint positions used for testing, set at $10^{\circ}$ intervals over the range from $20^{\circ}$ dorsiflexion to $30^{\circ}$ plantarflexion. An ankle joint angle of $0^{\circ}$ (neutral position) was defined as a $90^{\circ}$ angle between the fibula and fifth metatarsal bone. Following the familiarization sessions, MVC of the ankle plantarflexors and the fascicle length of the medial gastrocnemius (MG) were measured (Pre-measurement). The same measurements were performed after 4 weeks of intervention (Post-measurement), resulting in a total experimental period of 5 weeks. All measurements and analysis were implemented only at Kyoto University.

Subjects

Sixteen healthy women (age $21.8 \pm 1.5$ years), who were non-athletes and had not been involved in any regular stretching or resistance training, participated in this study. Subjects with a history of neuromuscular disease or musculoskeletal injury involving the lower limbs were excluded. The subjects were randomly assigned to the intervention group $(n=8)$ or to the control group $(n=8)$ using a computerized random number function in Microsoft Excel. All subjects were fully informed of the procedures and purpose of the study, which conformed to the Declaration of Helsinki. Written informed consent was obtained from all subjects. This study was approved by the ethics committee of 
1

2

3

4

5

6 Shirley, New York, USA.) with $1000 \mathrm{~Hz}$ sampling rate, at 6 ankle positions, set at $10^{\circ}$ intervals over

7 the range from $20^{\circ}$ dorsiflexion to $30^{\circ}$ plantarflexion, with the order of MVCs randomized across

Kyoto University Graduate School and the Faculty of Medicine (R-0216).

Measurements of MVC, the fascicle length and the muscle thickness

MVC was measured using a Biodex dynamometer (Biodex System 4, Biodex Medical Systems Inc., participants. A range of ankle joint angles (from $20^{\circ}$ dorsiflexion to $30^{\circ}$ plantarflexion) was determined by a range of motion that all subjects can exert force. For measurement, the ankle joint of the dominant leg was securely attached by velcro strap to the footplate of the dynamometer. Soft cloth was inserted between the velcro strap and instep to prevent unwanted movement of the ankle joint. The trunk and distal thigh were securely fixed by the dynamometer belts to keep the hip joint position of $80^{\circ}$ flexion and the knee joint in full extension. The subjects grasped horizontal bars attached to the dynamometer. MVC was exerted for 5 seconds (-s) at each of the 6 ankle joint angles, with more than 1 minute (-min) of rest provided between each MVC. The MVC peak torque over each 5-s trial was used for analysis.

The fascicle length of the MG was measured at the proximal 30\% of the lower leg length (1), using B-mode ultrasound imaging (LOGIQ e, General Electric, Duluth, GA, USA) with an 8-MHz linear 
1

2 Depth and Dynamic focus of the equipment settings were controlled to achieve a clear image of the muscle thickness and the fascicle length of the MG. The fascicle length was measured at each of the 6 test angles of the ankle and under three levels of activation—rest, 30\%MVC at the respective angles, and MVC—-for a total of 18 test conditions in randomized order. Three images of the fascicle were recorded for each condition. In measurement of the fascicle length at MVC, the images were preserved when exerted force displayed on the dynamometer monitor reached a plateau. The fascicle length was estimated from these images based on the methods which evaluated the distance along a straight line, between extension lines from the aponeurosis and the origin of the fascicle. (Figure 2). Ando et al. (2) demonstrated that this method is useful technique for estimating the fascicle length of quadriceps muscle. This method has been used to determine the fascicle length of the quadriceps muscle in a number of previous studies $(4,6,7)$. However, the reliability of the measurements of the MG has not been shown before. Therefore, the reliability of the measurements of MG was assessed at the 6 test ankle angles in a control group. A intraclass correlation coefficients (ICC 1.1) value higher than 0.75 is considered valid (12). ICC value for the fascicle length of the MG was valid at all ankle angles in both inter session (ICC > 0.9) that were measured by two images at pre-measurement, and inter day

17 (ICC > 0.75) that were measured by images at pre- and post-measurement. The fascicle length was measured for each condition using image processing software (ImageJ, version 1.48, National 
1 Institutes of Health, Bethesda, MD, USA). The mean value of all three images, obtained for each

2 condition, was used in the analysis. The outcome assessors were blinded to measurement conditions

3 (i.e., ankle joint position and level of activation). The fascicle length and the muscle thickness of MG

4 at rest with ankle position of $0^{\circ}$ were measured from the ultrasound imaging on the longitudinal plane

5 at baseline and after 4 weeks to examine morphological changes. The muscle thickness was also

6 measured to assess whether the improvement in muscle strength is due to morphologic changes such

7 as muscle hypertrophy. The muscle thickness of MG was measured at the proximal $30 \%$ of the lower

8 leg length. The muscle thickness was measured by measuring the line drawn perpendicular from the

9 surface to the deep aponeurosis. To accurately measure the muscle thickness without including non-

contractile tissue, the measurement between the inside edges of the aponeurosis was used. Previous

11 studies have shown the reliability of the ultrasound technique for measuring muscle thickness of the

MG. $(14,17)$

Training protocol

16 The intervention group performed resistance training at an intensity of 30\%MVC, 3 days per week,

17 for 4 weeks, using a Biodex dynamometer. The isometric resistance training protocol consisted of 3 
1

2 subjects could exert the target intensity at all training session. The training intensity of 30\%MVC was reassessed from MVC values measured after the 2-week intervention. Subjects in the control group did not receive any intervention.

5

$6 \quad$ Statistical analysis

7

8 Statistical analysis was performed using SPSS (version 22.0, SPSS Japan Inc., Tokyo, Japan).

9 Normality of the data was evaluated using a Shapiro-Wilk test. Group differences for characteristics and MVC of each ankle joint angle at baseline were assessed using an unpaired t-test. Two-way

11 repeated measures analysis of variance (ANOVA) using two factors (the ankle joint angle and the contraction level) was used to determine the differences in the fascicle length at the baseline due to

13 the ankle joint angle and the contraction level. When a significant main effect was observed,

14 Bonferroni's post hoc test was performed. In intervention group, paired t-test was used to determine the differences in the fascicle length between MVCs at each angle in Post-measurement, and at training condition (at $20^{\circ}$ plantarflexion with $30 \% \mathrm{MVC}$ ). The fascicle length at training condition was

17 calculated as the mean value of Pre- and Post-measurement to cancel the possible change in the fascicle length due to training. Split-plot ANOVA, using two factors (group $\times$ time), was used to analyze 
1 interaction effects for muscle strength. When a significant interaction was observed, a paired t-test

2 was used to determine the differences between the value at baseline and after 4 weeks in both groups.

3 The effect size (Cohen's d) of MVC changes in pre- and post-measurement was calculated employing

4 the methods used in the following study (16). Paired t-test was used to determine the differences of

5 the fascicle length and muscle thickness between at baseline and after 4 weeks. Differences were

6 considered to be statistically significant at an alpha level of 0.05 .

7

\section{$8 \quad$ RESULTS}

9

10 No subjects dropped out, and all subjects in the intervention group completed the training sessions.

11 Therefore, all data for all subjects in the intervention and control groups were entered in the analysis.

12 The characteristics of the subjects are shown in Table 1. There were no significant differences in

13 age, height, and body mass between subjects in the intervention and the control groups. Baseline MVC

of plantarflexion are shown in Table 2, again reported as the mean \pm SD. There were no significant

differences in MVC, across all 6 testing positions of the ankle joint angle, between intervention and control groups.

18 Differences in the fascicle length due to the ankle joint angle and the contraction level at baseline 
2 Figure 3 shows the fascicle length at rest, 30\%MVC, and MVC at each of the 6 test angles at

3 baseline ( $\mathrm{n}=16)$. Two-way repeated measures ANOVA, using two factors - the ankle joint angle and

4 the contraction level—indicated significant main effects for both the factors. The post-hoc analysis

5 showed significant differences in the fascicle length between all 6 test angles at each contraction level,

6 indicating that the fascicle length decreased with ankle plantarflexion. In addition, the post-hoc

7 analysis showed significant differences between all contraction levels, which shows that the fascicle

8 length decreases with increases in the contraction level at each ankle joint angle.

9

Effects of intervention on MVC

Effects of intervention on MVC, measured at each of the 6 test angles of ankle position, are shown

in Table 2. Split-plot ANOVA, using two factors (group $\times$ time), showed significant interactions at the not for the control group.

18 Effects of intervention on the muscle thickness and the fascicle length 
2 There were no significant differences in the fascicle length between baseline and after 4 weeks in

3 both groups (intervention group; baseline: $5.53 \pm 0.75 \mathrm{~cm}$, after: $5.56 \pm 0.78 \mathrm{~cm}$, control group;

baseline: $5.99 \pm 1.11 \mathrm{~cm}$, after: $5.73 \pm 1.36 \mathrm{~cm}$ ). There were also no significant differences in muscle

cm, after: $1.76 \pm 0.18 \mathrm{~cm}$, control group; baseline: $1.79 \pm 0.35 \mathrm{~cm}$, after: $1.74 \pm 0.27 \mathrm{~cm})$.

8 Comparison of the fascicle length during the training condition and MVC contraction

9

10 The fascicle lengths measured at $20^{\circ}, 10^{\circ}$ dorsiflexion, $0^{\circ}, 10^{\circ}, 20^{\circ}$ and $30^{\circ}$ plantarflexion with the

11 contraction level of MVC at Post-measurement were $4.39 \pm 1.29 \mathrm{~cm}, 3.54 \pm 0.90 \mathrm{~cm}, 3.30 \pm 1.16 \mathrm{~cm}$,

$2.77 \pm 0.68 \mathrm{~cm}, 2.48 \pm 0.43 \mathrm{~cm}$ and $2.33 \pm 0.34 \mathrm{~cm}$, respectively. The fascicle length at $20^{\circ}$ 


\section{DISCUSSION}

3 consistent with previous studies $(3,8)$. The fascicle length of the MG muscle at $20^{\circ}$ plantarflexion and at a contraction level of 30\%MVC (i.e. the training condition), was $2.95 \pm 0.35 \mathrm{~cm}$. This fascicle length was equivalent to the length measured at $0^{\circ}$ and $10^{\circ}$ plantarflexion at $\operatorname{MVC}\left(0^{\circ} ; 3.30 \pm 1.16 \mathrm{~cm}\right.$, $10^{\circ}$ plantarflexion. This result indicated that improvements in MVC were obtained at the same fascicle

17 length, rather than at the same joint angle, used during training, which consists with our hypothesis.

This is the first report demonstrating the influences of fascicle length during isometric training on improvement in muscle strength. The hypothesis of this study was that the effects of isometric training on MVC would be influenced by the muscle fascicle length rather than by the joint angle. Our results provided some evidence for this hypothesis, showing that MVC at $0^{\circ}$ and $10^{\circ}$ ankle plantarflexion increased after isometric training at $20^{\circ}$ plantarflexion, whereas no significant increase were observed in MVC at $20^{\circ}$ plantarflexion. In this study, the length of the muscle fascicle of the MG was defined as the length of the bundle of muscle fiber, estimated using ultrasound imaging. Our results showed that the fascicle length varied as a function of both the joint angle and the contraction level, which is $\left.10^{\circ} ; 2.77 \pm 0.68 \mathrm{~cm}\right)$. In this study, MVC improved not significantly at $20^{\circ}$ plantarflexion but at $0^{\circ}$ and 
1 2 the specificity of joint angle could also be considered that MVC improved specific to the fascicle MVC to be specific to the joint angle used during training. However, the results of these studies stating length. Since MVC was exerted during training in these studies, the relation of the fascicle length and the joint angle would be equal in training and MVC measurement. Therefore, the improvement found in the same joint angle would indicate improvement in the same fascicle length. the isometric contractions were performed for 3-s, which was shorter than contraction durations used in previous studies $(18,20,21)$. The rest period provided between sets was 2-min, which may be sufficient to recover from muscle fatigue. Therefore, we consider that in our study, the targeted training intensity of 30\%MVC was maintained and, therefore, that the fascicle length of the MG muscle was consistent during training. Previous studies $(18,20,21)$ showed that effects of high-intensity training on MVC were obtained at wider range of joint angles. In these previous studies $(18,20,21)$, the high resistance training intensity of $80 \% \mathrm{MVC}$ or $100 \% \mathrm{MVC}$ was performed with long isometric contraction or short rest time, such that the target intensity of $80 \% \mathrm{MVC}$ or $100 \% \mathrm{MVC}$ may not have been maintained. Under these conditions of isometric training with non-fixed intensities, the fascicle length could have varied, explaining the reported improvements in MVC over a range of joint angles.

17 In addition, it could be hypothesized that the muscle strength may increase over a wide range of joint angles if the magnitude of strength improvements was large. 
1

2 initial period of training (i.e., $<4$ weeks), followed by morphological adaptations in the muscles after

Improvement in muscle strength after resistance training depends on neural adaptations over the 6-8 weeks, which mainly contributes to the strength gains $(11,15)$. Our study showed no changes in morphological measurements such as the fascicle length or the muscle thickness after training. Therefore, the improvement in MVC after a 4-week intervention in this study may be influenced by neural adaptations, such as the increases in muscle activity of agonist muscles and decreases in antagonist coactivation (5). Further investigation is necessary to clarify the interaction between neural adaptation mechanisms and the effects of specificity in the fascicle length during training.

This study has some limitations. First, the fascicle length during training was not directly measured. Second, our study considered the fascicle length only for the MG muscle. Therefore, the influence of other plantar flexor muscles and the soleus and lateral gastrocnemius muscles, on measured improvements in MVC is unclear. That is to say, we cannot differentiate if improvements in MVC may

13 also be contributed by specificity in the fascicle length of the soleus or the lateral gastrocnemius muscles during training. Further research is required to clarify the effect of isometric training on MVC, considering the fascicle length of all muscles, which may contribute to strength gain. 

of the MG at the training position was similar to the fascicle length at the position that improved MVC, training at shortened muscle length may be suitable and safer for the elderly who cannot perform highintensity training or for patients who have restricted range of joint motion.

\section{Acknowledgments} that they have no conflict of interest.

\section{References}

1. Akagi $\mathrm{R}$ and Takahashi $\mathrm{H}$. Effect of a 5-week static stretching program on hardness of the gastrocnemius muscle. Scandinavian journal of medicine \& science in sports 24: 950-957, 2014.

2. Ando R, Taniguchi K, Saito A, Fujimiya M, Katayose M, and Akima H. Validity of fascicle length estimation in the vastus lateralis and vastus intermedius using ultrasonography. Journal of electromyography and kinesiology : official journal of the International Society of Electrophysiological Kinesiology 24: 214-220, 2014.

3. Arampatzis A, Mademli L, De Monte G, and Walsh M. Changes in fascicle length from rest to maximal voluntary contraction affect the assessment of voluntary activation. $J$ Biomech 40: 31933200, 2007.

4. Blazevich AJ, Cannavan D, Coleman DR, and Horne S. Influence of concentric and eccentric 
resistance training on architectural adaptation in human quadriceps muscles. $J$ Appl Physiol 103: 1565-1575, 2007.

5. Carolan B and Cafarelli E. Adaptations in coactivation after isometric resistance training. J Appl Physiol 73: 911-917, 1992.

6. Csapo R, Alegre LM, and Baron R. Time kinetics of acute changes in muscle architecture in response to resistance exercise. Journal of science and medicine in sport / Sports Medicine Australia 14: 270-274, 2011.

7. Ema R, Wakahara T, Mogi Y, Miyamoto N, Komatsu T, Kanehisa H, and Kawakami Y. In vivo measurement of human rectus femoris architecture by ultrasonography: validity and applicability. Clinical physiology and functional imaging 33: 267-273, 2013.

8. Fukunaga T, Ichinose Y, Ito M, Kawakami Y, and Fukashiro S. Determination of fascicle length and pennation in a contracting human muscle in vivo. J Appl Physiol 82: 354-358, 1997.

9. Kanehisa H and Miyashita M. Specificity of velocity in strength training. Eur J Appl Physiol Occup Physiol 52: 104-106, 1983.

10. Kitai TA and Sale DG. Specificity of joint angle in isometric training. Eur J Appl Physiol Occup Physiol 58: 744-748, 1989.

11. Kraemer WJ, Fleck SJ, and Evans WJ. Strength and power training: physiological mechanisms of adaptation. Exerc Sport Sci Rev 24: 363-397, 1996.

12. Lee J, Koh D, and Ong CN. Statistical evaluation of agreement between two methods for measuring a quantitative variable. Computers in biology and medicine 19: 61-70, 1989.

13. Lindh M. Increase of muscle strength from isometric quadriceps exercises at different knee angles. Scandinavian journal of rehabilitation medicine 11: 33-36, 1979.

14. Maganaris CN, Baltzopoulos V, and Sargeant AJ. In vivo measurements of the triceps surae complex architecture in man: implications for muscle function. The Journal of physiology 512 ( Pt 2): 603-614, 1998.

15. Moritani $\mathrm{T}$ and Devries HA. Neural factors versus hypertrophy in the time course of muscle strength gain. J Phys Med Rehabil 58: 115-130, 1979.

16. Nakagawa S and Cuthill IC. Effect size, confidence interval and statistical significance: a practical guide for biologists. Biological reviews of the Cambridge Philosophical Society 82: 591-605, 2007.

17. Narici MV, Binzoni T, Hiltbrand E, Fasel J, Terrier F, and Cerretelli P. In vivo human gastrocnemius architecture with changing joint angle at rest and during graded isometric contraction. The Journal of physiology 496 ( Pt 1): 287-297, 1996.

18. Rasch PJ and Pierson WR. One position versus multiple positions in isometric exercise. American journal of physical medicine 43: 10-12, 1964.

19. Thorstensson A, Hulten B, von Dobeln W, and Karlsson J. Effect of strength training on enzyme activities and fibre characteristics in human skeletal muscle. Acta Physiol Scand 96: 392-398, 1976. 
$120 . \quad$ Weir JP, Housh TJ, and Weir LL. Electromyographic evaluation of joint angle specificity and 2 cross-training after isometric training. J Appl Physiol 77: 197-201, 1994.

21. Weir JP, Housh TJ, Weir LL, and Johnson GO. Effects of unilateral isometric strength training on joint angle specificity and cross-training. Eur J Appl Physiol Occup Physiol 70: 337-343, 1995. 
1 Table 1 Characteristics of the participants

2

\begin{tabular}{crrrrr}
\hline & Intervention group $(\mathrm{n}=8)$ & Control group(n=8) & $p$-valüe \\
\hline Age (years) & 21.75 & \pm 0.71 & $21.88 \quad \pm 2.10$ & 0.88 \\
Height $(\mathrm{cm})$ & 159.13 & \pm 5.41 & $158.13 \quad \pm 3.50$ & $0.6 \bar{p}$ \\
Body mass $(\mathrm{kg})$ & 50.63 & \pm 5.32 & $49.13 \pm 4.05$ & 0.56 \\
\hline
\end{tabular}

8 Values are expressed as mean \pm SD (standard deviation)

9 
1 Table 2 Effects of intervention on MVC at each ankle joint angle

\begin{tabular}{|c|c|c|c|c|c|c|c|c|c|c|c|}
\hline \multirow{3}{*}{$\frac{\text { ankle joint angle }}{\text { Dorsiflexion } 20^{\circ}}$} & \multicolumn{6}{|c|}{ intervention group $(\mathrm{n}=8)$} & \multicolumn{5}{|c|}{ control group $(\mathrm{n}=8)$} \\
\hline & \multicolumn{2}{|c|}{ Pre (Nm) } & \multicolumn{3}{|c|}{ Post (Nm) } & \multirow{2}{*}{$\frac{\text { Effect size }(95 \% \mathrm{CI})}{0.53(-0.47-1.53)}$} & \multicolumn{2}{|c|}{ Pre (Nm) } & \multicolumn{2}{|c|}{ Post (Nm) } & \multirow{2}{*}{$\frac{\text { Effect size }(95 \% \mathrm{CI})}{0.06(-0.92-1.04)}$} \\
\hline & 112.55 & \pm 37.79 & 131.09 & \pm & 31.92 & & 116.08 & \pm 40.98 & 118.53 & \pm 42.26 & \\
\hline Dorsiflexion $10^{\circ}$ & 101.88 & \pm 30.15 & 119.24 & \pm & 27.26 & $0.60(-0.40-1.61)$ & 103.28 & \pm 35.82 & 104.76 & \pm 36.09 & $0.04(-0.94-1.02)$ \\
\hline $0^{\circ * *}$ & 81.26 & \pm 26.52 & 105.08 & \pm & 21.56 & $0.99(-0.05-2.02)$ & 91.50 & \pm 24.60 & 90.78 & \pm 29.04 & $-0.03(-1.01-0.95)$ \\
\hline Plantarflexion $10^{\circ *}$ & 62.96 & \pm 23.55 & 81.29 & \pm & 20.30 & $0.83(-0.19-1.86)$ & 71.50 & \pm 21.06 & 70.15 & \pm 21.06 & $-0.06(-1.04-0.92)$ \\
\hline Plantarflexion $20^{\circ}$ & 50.23 & \pm 21.14 & 62.24 & \pm & 20.43 & $0.58(-0.42-1.58)$ & 51.51 & \pm 16.30 & 53.76 & \pm 17.44 & $0.13(-0.85-1.11)$ \\
\hline Plantarflexion $30^{\circ}$ & 31.84 & \pm 14.70 & 42.74 & \pm & 16.60 & $0.70(-0.31-1.70)$ & 34.03 & \pm 15.96 & 36.38 & \pm 15.32 & $0.15(-0.83-1.13)$ \\
\hline
\end{tabular}

2 Values are expressed as mean \pm SD (standard deviation)

$3 \mathrm{CI}=$ confidence interval

4 Effect size is Cohen’s d. d = 0.2, 0.5, 0.8 were considered "small,” “medium,” and "large” effects, respectively.

$5 \quad *$; significant interaction between time (Pre and Post) and groups (intervention and control groups)

$6 \quad * * P<0.01, * P<0.05$ 
$1 \quad$ Fig. 1 Flowchart of this study

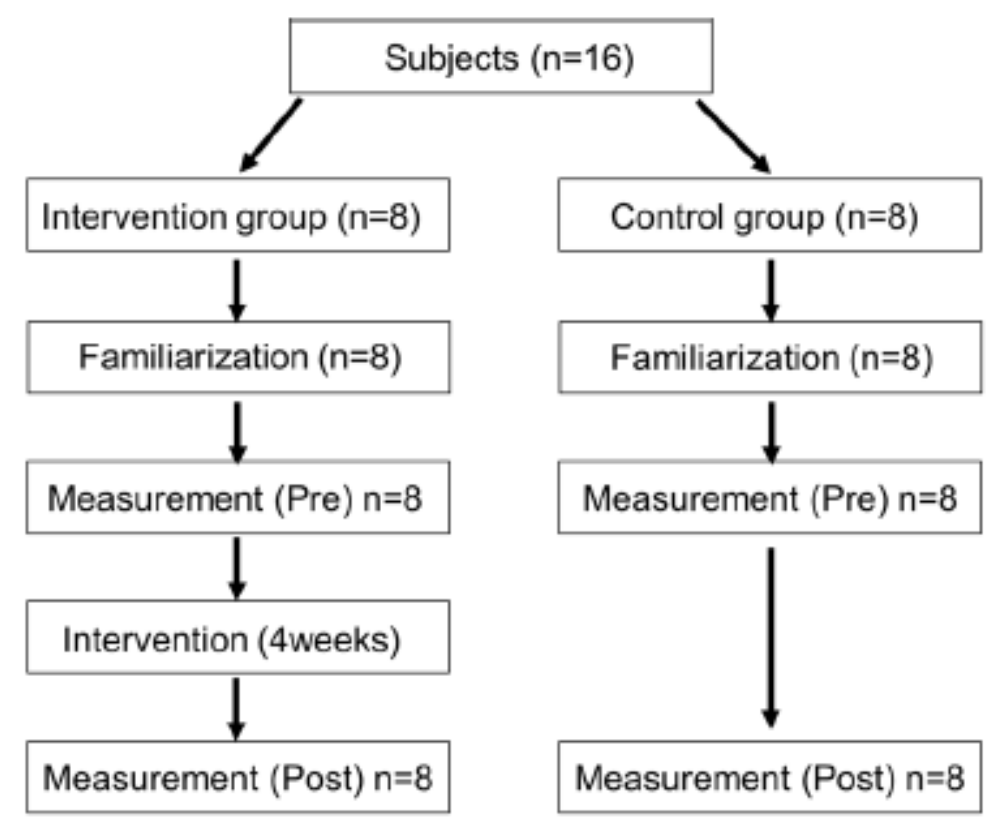

2 
$1 \quad$ Fig. 2 Estimation of fascicle length

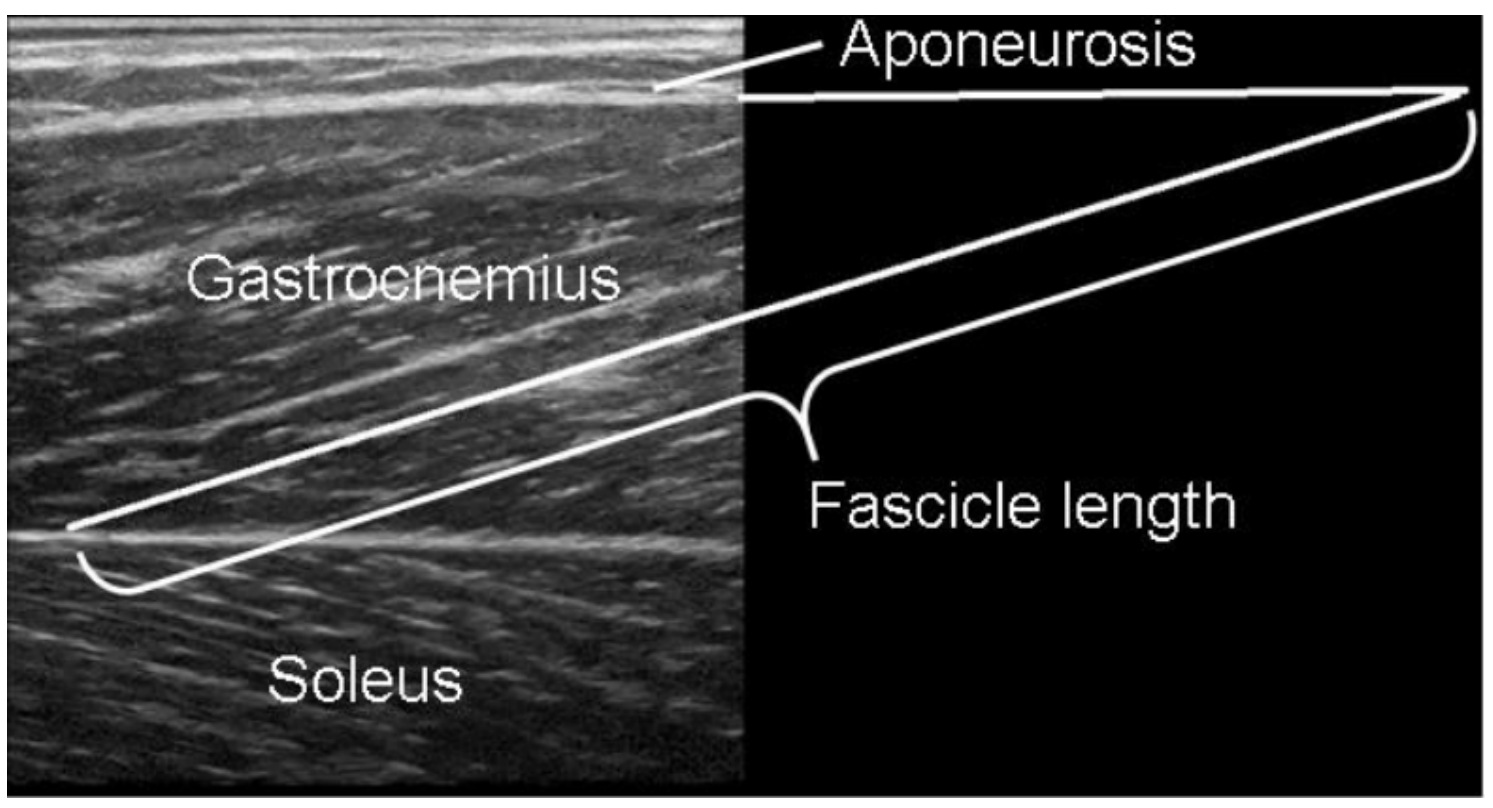

The fascicle length of the MG was defined as a distance in a straight line between extension lines from aponeurosis and fascicle origin. 
$1 \quad$ Fig.3 Fascicle length in both groups at baseline

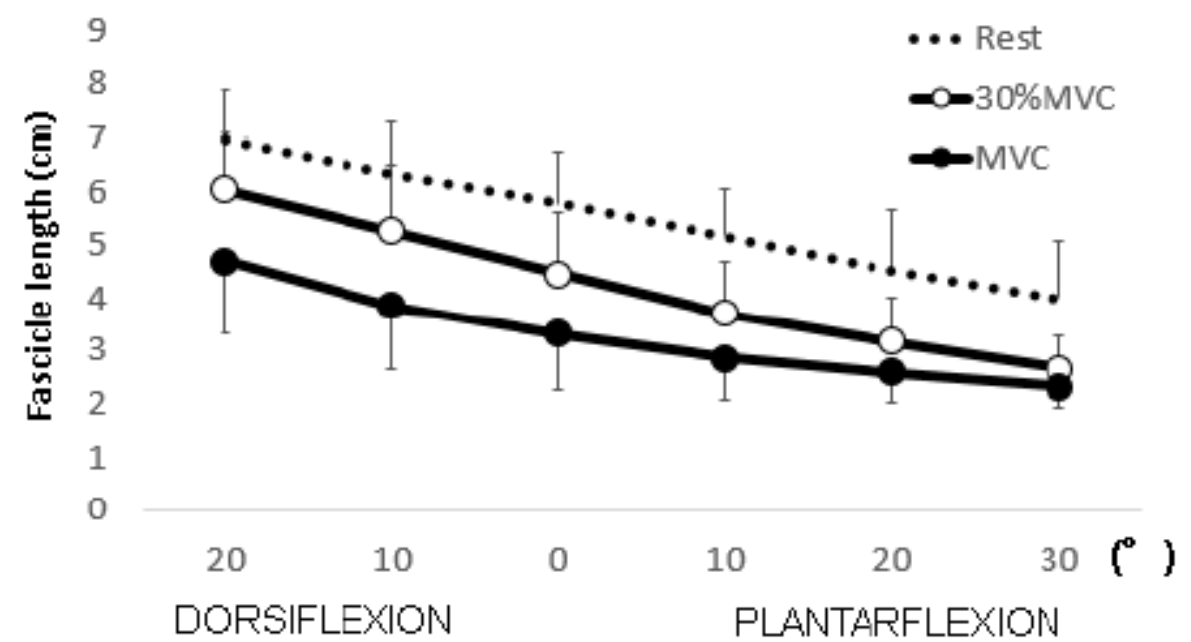

3 An open circle indicates 30\%MVC, and a filled circle indicates MVC values. At all contraction

4 levels, the fascicle length decreases toward plantarflexion. At the same ankle angle, fascicle length 5 decreases toward MVC. 